\title{
Neuro-Oncology: Current Concepts and Emerging Therapeutics
}

\author{
Burt Nabors $^{1}$ • John Laterra ${ }^{2}$ (D)
}

Published online: 10 April 2017

(C) The American Society for Experimental NeuroTherapeutics, Inc. 2017

Having the opportunity to serve as Guest Editors of this issue of Neurotherapeutics on neuro-oncology forced by design a critical consideration of current knowledge, the most impactful advances in evidence-based diagnostics and therapeutics, and the most promising basic and clinical research directions. Neuro-oncology is expansive from almost all perspectives. There are over 100 distinct pediatric and adult primary brain tumors and the number of pathological entities is increasing with the implementation of genomic and molecular diagnostic subclassification. Beyond the realm of primary brain tumors are the far more common though less diverse brain metastases and the rare but increasingly recognized paraneoplastic syndromes. We have chosen from this wealth of potential topics to focus with some exceptions on the most common and most characteristically life-altering primary brain tumors in adults and children, glioma and medulloblastoma - arguably the most clinically challenging and biologically interesting of central nervous system (CNS) malignancies.

A brief retrospective offers a valuable perspective on how the discipline of neuro-oncology has evolved and advanced over the past few decades. Prior to the mid-1990s, the field of neuro-oncology consisted of individual or small groups of pioneering clinicians and disease-oriented scientists working on seemingly intractable problems in relative isolation compared with today. The field began to change dramatically with the establishment in 1993 of the first National Cancer Institute (NCI)-funded multi-institutional adult brain tumor clinical

John Laterra

jlaterr1@jhmi.edu

1 University of Alabama School of Medicine, Birmingham, AL, USA

2 Johns Hopkins School of Medicine, Baltimore, MD, USA consortia devoted to discovering new therapies via phase I/II clinical trials, the founding of the Society for Neuro-Oncology (SNO), and SNO's first scientific meeting held in Santa Fe, New Mexico in 1996. The Pediatric Brain Tumor Consortium was established by the NCI in 1999 and the three original adult NCI consortia have since consolidated to the Adult Brain Tumor Consortium, a single 11-member institutional collaborative group. Since its inception, SNO membership and meeting participants have expanded from about 330 at founding to now over 2000 representing over 40 countries. The first issue of the official journal of the SNO, NeuroOncology, was published in January 1999 and now represents the premier subspecialty venue for communicating basic, translational, and clinical neuro-oncology research. A complementary SNO publication, Neuro-Oncology Practice, focusing on quality of life and clinical care, premiered in March 2014. In 2007, Neuro-Oncology became an official United Council for Neurological Subspecialties boardcertifiable subspecialty resulting in the formalization of neuro-oncology training standards at 27 currently certified academic centers. These organizational developments spurring dramatic increases in brain cancer research and clinical care advances predict a future of more rapid discoveries and more effective treatments.

Brain cancer therapy has evolved over the past couple of decades into an evidence-based discipline driven by randomized and controlled clinical trials. Radiation therapy continues to offer convincing first-line benefit following surgical resection for malignant brain tumors. The addition of chemotherapy to the treatment of newly diagnosed glioblastoma and other malignant gliomas failed to realize a significant clinical benefit until the randomized European Organisation for Research and Treatment of Cancer (EORTC) controlled trial comparing temozolomide plus standard fractionated radiation to radiation therapy alone in the treatment of newly diagnosed 
glioblastoma. The results demonstrated an advantage in overall survival, progression-free survival, and percentage of patients alive at 2 years [1]. The advantage persisted through 5 years of follow-up [2]. In addition, this effort implicated a tumor-specific DNA repair enzyme, methyl-guanine methyl transferase, as a mediator of temozolomide resistance. Specifically, overall survival following combination chemotherapy and radiation was found to be significantly longer for patients with tumor $M G M T$ gene silencing through promoter methylation than for patients with unmethylated $M G M T$ (21.7 months vs 12.7 months). Resulting from this advance, neuro-oncologists are now caring for an unprecedented number of long-term survivors with glioblastoma. Recent randomized controlled studies have also revealed remarkable survival advantages by implementing early chemotherapy for patients with anaplastic oligodendroglioma. Two independent studies, one in the USA [Radiation Therapy Oncology Group (RTOG) 9402] and a European counterpart (EORTC 26951) examined the role of the chemotherapy regimen PCV (procarbazine, lomustine, and vincristine) either before (neoadjuvant) or after (adjuvant) radiation therapy in newly diagnosed anaplastic oligodendroglioma. Both studies demonstrated similar prolongations in median overall survival from 7 years to about 15 years, in response to combination therapy. These studies also found that this truly remarkable survival advantage was limited to tumors with $1 \mathrm{P}$ and $19 \mathrm{Q}$ loss of heterozygosity (codeletion), now recognized to be the genomic hallmark of oligodendroglioma $[3,4]$. One is hard-pressed to find recent clinical trials demonstrating such impressive survival advantages in any other solid malignancy.

Efforts to incorporate targeted and biological therapy into the initial treatment of glioblastoma have been less successful. The most extensively evaluated has been the vascular endothelial growth factor (VEGF) neutralizing antibody bevacizumab (Avastin). The biological phenomenon of tumor-associated blood vessel proliferation (angiogenesis) is recognized as a significant sequela of high intratumoral VEGF production, a suspected driver of gliomagenesis and definite contributor to neurological dysfunction due to blood-brain barrier dysfunction and associated cerebral edema. Unfortunately, adding bevacizumab to standard radiation and temozolomide for newly diagnosed glioblastoma yielded no survival advantage in two randomized, controlled, and blinded clinical trials $[5,6]$. Subset analysis and clinical experience suggests the existence of patient subsets that may benefit from VEGF-targeted therapy and continued efforts to identify these and understand the molecular driving events are ongoing. Currently, the benefit of bevacizumab is limited to patients with recurrent malignant glioma based on improved quality of life and prolonged progression-free survival as evaluated by magnetic resonance imaging (MRI) [7]. The significance of these radiographic and clinical responses to bevacizumab remains controversial as bevacizumab has the potential to normalize the disrupted tumor-associated blood-brain barrier, reduce cerebral edema, and thereby improve symptoms in the absence of a true antitumor effect (i.e., pseudo-response). Pseudo-response and pseudo-progression, the well-documented worsening of MRI in response to treatment-induced blood-brain barrier disruption in the absence of true tumor progression, are major obstacles to clinical decision making within and outside of clinical trial therapy. Improving the specificity of MRI, positron emission spectroscopy, and other nonsurgical diagnostics is critical to the future of clinical neuro-oncology that is so highly dependent upon image-based decision making.

Progress in treating glioblastoma, the most common and aggressive malignant brain tumor, is being made but too slowly and too incrementally. There remains continued frustration over the pace at which substantive developments have translated to improving patient survival. In contrast to this is the excitement and promise stemming from the fact that the most common adult and pediatric malignant brain tumors now represent some of the most extensively studied cancers at the molecular, genetic, and epigenetic levels, owing, in great part, to the NCI-funded Cancer Genome Atlas Project initiated in 2005 and comparably modeled collaborative efforts in medulloblastoma and other less common tumor types [8]. This has led to a deeper understanding of the molecular basis of brain cancer heterogeneity and a foundation for more advanced evidenced-based clinical decision making and personalized targeted therapeutics. Positive developments include how medulloblastoma molecular subtyping has started to impact treatment regimens, and the potential therapeutic efficacy of BRAF inhibitor therapy in BRAFV600E mutated brain tumors [9]. These significant advances in defining the molecular underpinnings of CNS cancers and the contributions these events make to the disease biology, as well as the ability to subclassify malignancies based on genomics are leading to improved therapeutic recommendations.

The current environment of translating molecular and biological discoveries into meaningful advances for patients is dependent upon early-phase clinical trials, which emphasize defining and identifying specific targets and quantifying target effects within tumor tissue. The integration of biological endpoints into clinical development remains challenging. Numerous factors from the identification of eligible patients, involvement of multiple clinical and scientific disciplines, and assay development create a bottleneck to the timely translation of laboratory findings to patients. Solutions involve multidisciplinary research teams, engagement and participation of laboratory scientists in clinical research, and advanced understanding of targets in the tumor microenvironment.

Despite major setbacks in large randomized clinical trials, the future for patients with CNS cancers is improving. As can be seen in this issue of Neurotherapeutics, advances are occurring across disciplines. Among the most exciting and of immediate clinical utility include the subclassification of 
glioma and medulloblastoma with incorporation of the new World Health Organization guidelines. This progress is reviewed and discussed by Howard Colman and Scott Pomeroy. David Eisenstat addresses epigenetic pathways in pediatric high-grade glioma with an emphasis on their role in disease progression and potential as therapeutic targets. Building upon these molecular insights, Stephen Dombrowski and David James explore the unique nature of brain cancer stem cells, and the utility and role of contemporary animal models for preclinical therapeutic testing. We have invited leading neuro-oncology experts to address the deployment of experimental therapeutics to patients. These include approaches for delivering chemotherapy and molecular therapeutics directly into tumors using neurosurgical guidance such as convention-enhanced delivery, presented by Jeffrey Bruce, and oncolytic virotherapies, presented by James Markert. The rapidly expanding field of immunotherapy and immune checkpoint blockade is presented by Michael Lim, and Sherise Fergerson addresses current experience with tumor vaccines and strategies for future improvements. Drs. Tracy Batchelor and Shiao-Pei Weathers offer insights into new directions for antiangiogenic agents and strategies for implementing personalized targeted therapy. A consistent challenge for both pediatric and adult neuro-oncology is the optimal use and interpretation of neuroimaging, especially in diagnosing disease response during clinical trials. Ben Ellingson provides recommendations on the role of MRI and the selection of criteria to standardize this process across the field. The neuro-oncology community is at the forefront in caring for patients with inherited tumor syndromes. Representing this is Verena Staedtke's examination of current concepts and research related to peripheral nerve malignancies in patients with the common inherited cancer predisposition syndrome neurofibromatosis 1 .
We are pleased to present this issue of Neurotherapeutics focused on the current and most promising developments in neuro-oncology. We hope readers find the volume highly informative with contributing papers providing rich overviews, perspectives, and future visions.

\section{References}

1. Stupp R, Mason WP, van den Bent MJ, et al. (2005). Radiotherapy plus concomitant and adjuvant temozolomide for glioblastoma. $\mathrm{N}$ Engl J Med 352: 987-996.

2. Stupp R, Hegi ME, Mason WP, et al. (2009). Effects of radiotherapy with concomitant and adjuvant temozolomide versus radiotherapy alone on survival in glioblastoma in a randomised phase III study: 5-year analysis of the EORTC-NCIC trial. Lancet Oncol 10: 459466.

3. Cairncross G, Wang M, Shaw E, et al. (2013). Phase III trial of chemoradiotherapy for anaplastic oligodendroglioma: long-term results of RTOG 9402. J Clin Oncol 31: 337-343.

4. van den Bent MJ, Brandes AA, Taphoorn MJ, et al. (2013). Adjuvant procarbazine, lomustine, and vincristine chemotherapy in newly diagnosed anaplastic oligodendroglioma: long-term follow-up of EORTC brain tumor group study 26951. J Clin Oncol 31: 344-350.

5. Gilbert MR, Sulman EP, Mehta MP (2014). Bevacizumab for newly diagnosed glioblastoma. N Engl J Med 370: 2048-2049.

6. Chinot OL, Wick W, Mason W, et al. (2014). Bevacizumab plus radiotherapy-temozolomide for newly diagnosed glioblastoma. $\mathrm{N}$ Engl J Med 370: 709-722.

7. Cohen MH, Shen YL, Keegan P, et al. (2009). FDA drug approval summary: bevacizumab (Avastin) as treatment of recurrent glioblastoma multiforme. Oncologist 14: 1131-1138.

8. The Cancer Genome Atlas Research Network (2008). Comprehensive genomic characterization defines human glioblastoma genes and core pathways. Nature 455:1061-1068.

9. Brown NF, Carter T and Mulholland P (2017). Dabrafenib in BRAFV600E-mutated anaplastic pleomorphic xanthoastrocytoma. CNS Oncol 6:5-9. 